\title{
Large deformation analysis of Euler-Bernoulli beamshell under own weight based on HAM
}

Research Article

\author{
Abdol Rasoul Sohouli ${ }^{1}$, Amin Kimiaeifar²*, Afshin Mohsenzadeh ${ }^{2}$, Saeed Reza Mohebpour ${ }^{3}$ \\ 1 Babol University of Technology, Department of Mechanical Engineering P. O. Box 484, Babol, Iran, \\ 2 Department of Mechanical and Manufacturing Engineering, Aalborg University, \\ Pontoppidanstraede 101, DK-9220 Aalborg East, Denmark, \\ 3 Department of Mechanical Engineering, Persian Gulf University, Bushehr, Iran
}

Received 23 March 2011 ; accepted 7 July 2011

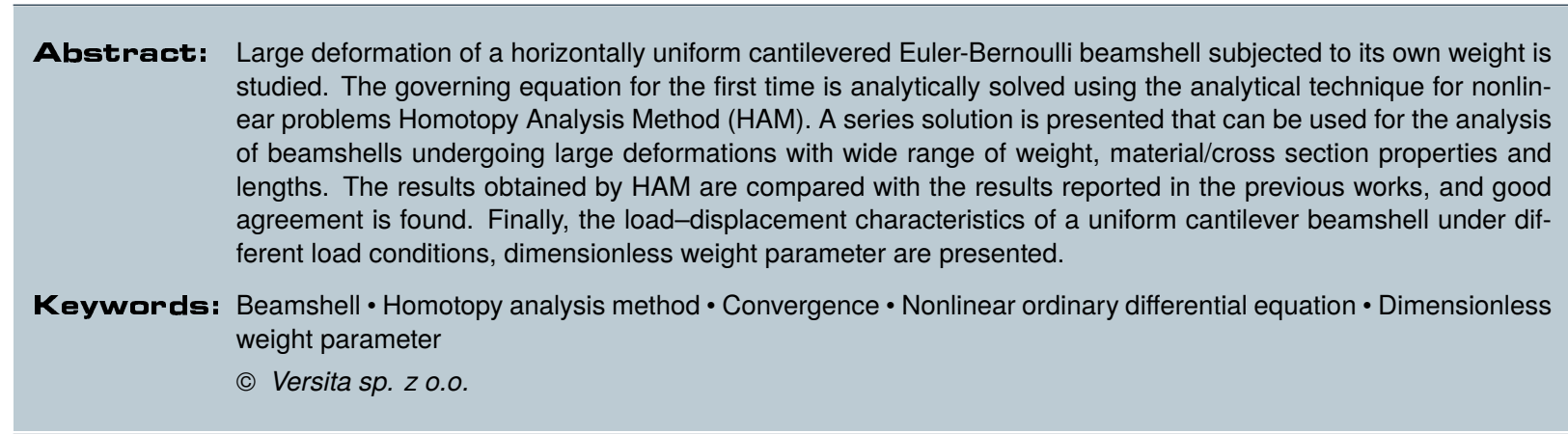

\section{Introduction}

Due to the reduction of weight, material and cost of structures, beamshells and thin-walled beams are extensively used in aero space, automotive and aircraft industries. Such flexible structures can undergo large displacement and rotations without exceeding their elastic limits. To understand the behavior of these flexible structures and to evaluate their elastic limits many different formulations and numerical procedures have been proposed for displacement and rotation analysis of beamshells [1].

Hummel and Morton [2], solved this problem in terms of a power series, which subsequently was numerically solved

*E-mail: akf@me.aau.dk by Bickley [3]; with some corrections, Rohde [4] improved the accuracy. Wang [5] noted that the first integral of governing equation of the problem was incorrect because it was valid only if $\bar{x}$ (position) is a linear function of $s$ (independent dimensionless parameter). A related problem that of the "stiff" catenary - is to determine the shape of a beamshell under its own weight if its two ends are built into piers less than a distance $L$ apart, which are not necessarily at the same height. An approximate solution in which the effect of bending stiffness is treated as a small, singular perturbation of the classic catenary problem may be found in van der Heijden [6], and Simmonds and Mann [7].

Other similar works have been done on the analysis of problems involving large deflections and rotations. Holden [8] proposed a numerical approach to the problem of finite 
deflections of a linear elastic cantilever beam with uniformly distributed load using a fourth order Runge-Kutta. Wang [9-11] presented a number of problems involving large deflections of linear elastic beams under various external loads as well as own-weight by adopting a perturbation method and numerical integration.

Many solid mechanics problems such as beamshells are inherently nonlinear. Except for a limited number of these problems, most of them do not have analytical solution. Therefore, these nonlinear equations should be solved using other methods. Some of them are solved numerically and some are solved using analytical methods, extended from perturbation technique [8]. In numerical methods, stability and convergence should be considered to ensure realistic results. In the analytical perturbation method, a small parameter should be exerted in the equation; the determination and exertion of this small parameter into the equation are deficiencies of this method.

One of the semi-exact methods which does not need small/large parameters is the Homotopy Analysis Method (HAM), first proposed by Liao $[12,13]$. This method has already been successfully applied to many problems in solid mechanics such as large deformation of beams, nonlinear vibration and oscillations and also nonlinear $\mathrm{dy}$ namics [14-20]. In this method, the convergent region can be adjusted and controlled, which is the most important feature of this technique in contrast to other techniques such as homotopy perturbation method, volitional iteration method and energy balanced method.

In the present work, HAM has been applied to obtain analytical solutions for the boundary value problems constituted by the nonlinear ordinary differential equation governing of a cantilever beamshell under large deflection, together with the associated boundary conditions. To show the accuracy of the proposed methodology, the results obtained from HAM for different cases are compared with results obtained in previous studies. In addition to establishing the robustness of the solution, the convergence region for different values of load and materials/cross section properties is presented. Finally, the influence of a dimensionless weight parameter on the slope and tip-deformed configuration are discussed.

\section{Governing Equation}

From the equation of motion it is obtained that [1]:

$$
\left\{\begin{array}{l}
F^{\prime}+P-m \dot{v}=0 \\
M^{\prime}+m . F+l-l \dot{\omega}=0
\end{array}\right.
$$

Where $P$ and $F$ can be represented in the alternative forms as below:

$$
\left\{\begin{array}{l}
P=P_{x} e_{x}+P_{y} e_{y} \\
F=H e_{x}+V e_{y}
\end{array}\right.
$$

From Eqs. 1 and 2:

$$
\begin{gathered}
H^{\prime}+P_{x}+0, \quad V^{\prime}+P_{y}=0 \\
M^{\prime}+\lambda(V \cos \beta-H \sin \beta)+l=0
\end{gathered}
$$

It is clear that, when the beam-shell is under small deformation, for simplification it can be assumed that $\sin \beta=\beta$ and $\cos \beta=1$. The advantage of this form the equilibrium equation is obvious from the integration of Eq. 3:

$$
\left\{\begin{array}{l}
H=-\int P_{x} d \sigma \\
V=-\int P_{y} d \sigma
\end{array}\right.
$$

And the reduction of Eq. 4 to:

$$
M^{\prime}=\lambda\left(\left\{\int P_{y} d \sigma \cos \beta\right\}-\left\{\int P_{x} d \sigma \sin \beta\right\}\right)-l
$$

Since for the present problem $H=0$ and $V=-m g(L-\sigma)$, Eq. 4 is further reduced to:

$$
M^{\prime}-m g(L-\sigma) \cos \beta=0, \quad M^{\prime}=\frac{L^{2}}{E_{b} h^{3}} \ddot{\beta}
$$

Here the introduction of a dimensionless distance and weight parameter is made according to:

$$
s=\frac{\sigma}{L}, \quad \mu=\frac{m g L^{3}}{E_{b} h^{3}}
$$

By substituting Eq. 8 into Eq. 7, and adjoining the boundary conditions where the angle of rotation is zero at the fixed end, and the moment is zero at the free end, a twopoint boundary value problem is obtained as the governing equation (see Fig. 1):

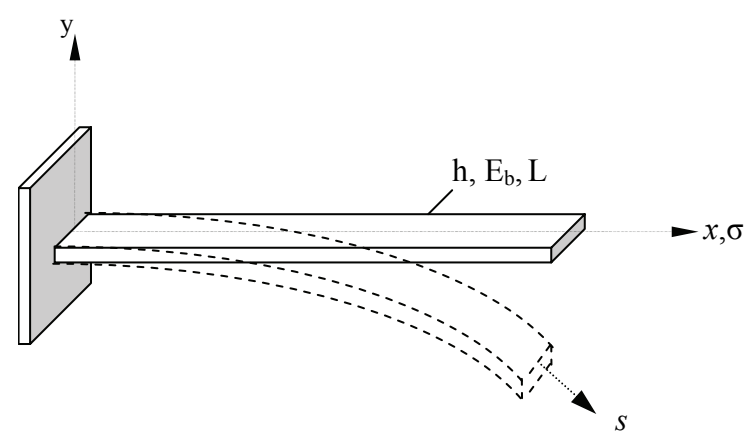

Figure 1. The geometry and boundary conditions of a beamshell. 


$$
\ddot{\beta}-\mu(1-s) \cos \beta=0, \quad \beta(0)=\dot{\beta}(1)=0 \quad \dot{\beta}=\frac{d \beta}{d s}
$$

The tip position of the deformed beamshell is found from:

$$
\begin{aligned}
\bar{y}(L)= & L\left(\left\{\int_{0}^{1} \cos (\beta(s, \mu)) d s\right\} e_{x}\right. \\
& \left.+\left\{\int \sin (\beta(s, \mu)) d s\right\} e_{y}\right)
\end{aligned}
$$

\section{Application of the Homotopy Analysis Method}

Consider the governing equation of a beamshell expressed by Eq. 9 . The nonlinear operator is defined as follows:

$$
N[\beta(s ; q)]=\frac{\partial^{2} \beta(s ; q)}{\partial s^{2}}-\mu(1-s) \cos (\beta(s ; q))
$$

The expansion of $\cos (\beta(s ; q))$ in Taylor series is:

$$
\cos (\beta(s ; q)) \approx 1-\frac{1}{2} \beta^{2}(s ; q)+\frac{1}{24} \beta^{4}(s ; q)
$$

Substituting Eq. 12 into Eq. 11, gives:

$$
\begin{aligned}
N[\beta(s ; q)]= & \frac{\partial^{2} \beta(s ; q)}{\partial s^{2}}-\mu(1-s)\left(1-\frac{1}{2} \beta^{2}(s ; q)\right. \\
& \left.+\frac{1}{24} \beta^{4}(s ; q)\right)
\end{aligned}
$$

Where $q \in[0,1]$ is the embedding parameter, and $\hbar \neq$ 0 is a nonzero auxiliary parameter. As the embedding parameter increases from 0 to $1, U(s ; q)$ varies from the initial guess $U_{o}(s)$ to the exact solution $U(s)$ :

$$
\beta(s ; 0)=U_{0}(s), \quad \beta(s ; 1)=U(s)
$$

$U(s ; q)$ can be expanded in Taylor series with respect to $q$ :

$$
\beta(s ; q)=U_{0}(s)+\sum_{m=1}^{\infty} U_{m}(s) q^{m}
$$

Where

$$
U_{m}(s)=\left.\frac{1}{m !} \frac{\partial^{m} \beta(s ; q)}{\partial q^{m}}\right|_{q=0}
$$

Homotopy analysis method can be expressed by many different base functions [13], according to the governing equation; it is straightforward to use a set of base functions:

$$
\left\{s^{n} \mid n=0,1,2,3, \ldots\right\}
$$

In the form:

$$
U(s)=\sum_{n=0}^{\infty} b_{n} s^{n}
$$

Such $b_{n}$ that is a coefficient to be determined. Besides determining a set of base functions, the auxiliary function $H(s)$, initial approximationand $U_{0}(s)$ the auxiliary linear operatormust $£$ be chosen in such a way that all solutions of the corresponding high-order deformation equations exist and can be expressed by this set of base functions, and the other expressions such as $s^{n} \sin (m s)$ must be avoided. This conforms to the so-called rule of solution expression [13].

Here a linear operator is chosen, as below:

$$
£[\beta(s ; q)]=\frac{\partial^{2} \beta(s ; q)}{\partial s^{2}}
$$

With the property

$$
£\left[c_{1} s+c_{2}\right]=0
$$

Where $c_{1}, c_{2}$ are integral constants. Under the rule of solution expression, the initial condition can be chosen as:

$$
U_{0}(s)=0
$$

The zero ${ }^{\text {th }}$ order deformation equation is:

$$
\begin{aligned}
& (1-q) L\left(\left[\beta(s ; q)-U_{0}(s)\right]=q h H(s) N[\beta(s ; q)]\right. \\
& \beta(0 ; q)=0, \quad \frac{\partial \beta(1 ; q)}{\partial s}=0
\end{aligned}
$$

According to the rule of solution expression denoted by Eq. 18 and from Eq. 22, the auxiliary function can be chosen as follows:

$$
H(s)=s^{k}
$$

Differentiating Eq. $22 \mathrm{~m}$ times with respect to the embedding parameter $q$, then setting $q=0$ and finally dividing them by $m$ !, from Eq.13 and 18 we have the so-called $m^{\text {th }}$-order deformation equation for $m \geq 1$ :

$$
\begin{aligned}
& \begin{array}{ll}
U_{m}(s)=\chi_{m} U_{m-1}(s) & +\hbar \int_{0}^{s} \int_{0}^{\tau} H(s) R_{m}\left(\vec{U}_{m-1}\right) d s d \tau \\
& +c_{1} s+c_{2}
\end{array} \\
& U_{m}(0)=U_{m}^{\prime}(1)=0
\end{aligned}
$$

Where: 


$$
\begin{aligned}
R_{m}\left(\vec{U}_{m-1}\right)= & \left(\frac{d^{2}}{d q^{2}} U_{m-1}(s)\right)-\left(1-\chi_{m}\right) \mu+\left(1-\chi_{m}\right) \mu s+\left(\frac{\mu}{2}-\frac{\mu}{2}\right)\left(\sum_{z=0}^{m-1} U_{z}(s) U_{m-1-z}(s)\right) \\
& +\left(\frac{\mu s}{24}-\frac{\mu}{24}\right)\left(\sum_{n=0}^{m-1} \sum_{z=0}^{n} U_{z}(s) U_{n-z}(s) \sum_{j=0}^{m-1-n} U_{j}(s) U_{m-1-n-j}(s)\right)
\end{aligned}
$$

$$
\chi_{m}= \begin{cases}0, & m \leq 1 \\ 1, & m>1\end{cases}
$$

$H(s)$ can be chosen uniquely under the rule of solution expression and rule of coefficient ergodicity. It can be shown that when $(k=2 n \mid n=1,2,3, \ldots))$, the terms $s^{5}$ up to $s^{2 n+1}$ always disappear in the solution expression of $U_{m}(s)$, so that the coefficients of the terms $s^{5}$ up to $s^{2 n+1}$ are always zero and can not be modified even if the order of approximation tends to infinity. This disobeys the so-called rule of coefficient ergodicity, which was expressed by Liao [13]. If $(k=2 n+1 \mid n=1,2,3, \ldots))$ then bases like $s^{2 m+1}$ will disappear, which disobeys the rule of solution expression and the rule of coefficient ergodicity. On the other hand when $k=-1,-2,-3$, the terms $s \ln s, \ln s$ will appear. Consequently the corresponding auxiliary function was determined uniquely as $H(s)=1$, where $k=1$. It is successively obtained:

$$
\begin{aligned}
U_{0}(s)= & 0 \\
U_{1}(s)= & \frac{1}{6} h \mu s^{3}-\frac{1}{2} h \mu s^{2}+\frac{1}{2} h \mu s \\
U_{2}(s)= & \left(\frac{1}{6} h^{2} \mu+\frac{1}{6} h \mu\right) s^{3}+\left(-\frac{1}{2} h^{2} \mu-\frac{1}{2} h \mu\right) s^{2}+\left(\frac{1}{2} h^{2} \mu=\frac{1}{2} h \mu\right) s \\
U_{3}(s)= & -\frac{1}{5184} \mu^{3} h^{3} s^{9}+\frac{1}{576} \mu^{3} h^{3} s^{8}-\frac{1}{144} \mu^{3} h^{3} s^{7}+\frac{11}{720} \mu^{3} h^{3} s^{6}-\frac{3}{160} \mu^{3} h^{3} s^{5}+\frac{1}{96} \mu^{3} h^{3} s^{4} \\
& +\left(\frac{1}{6} \mu h+\frac{1}{6} h^{3} \mu+\frac{1}{3} h^{2} \mu\right) s^{3}+\left(-\frac{1}{2} \mu h-\frac{1}{2} \mu h^{3}-h^{2} \mu\right) s^{2}+\left(h^{2} \mu-\frac{1}{320} \mu^{3} h^{3}+\frac{1}{2} h^{3}+\frac{1}{2} h^{3} \mu+\frac{1}{2} h \mu\right) s
\end{aligned}
$$

Solution was developed up to 20th order of approximation of $U(s)$ (see Fig.2 and Fig.3 and Table (1) and (2)).

\section{Convergence of HAM solution}

It should be pointed out that the auxiliary parameter $\hbar$, as pointed out by Liao [9], controls the convergence and accuracy of the solution series. In order to define a region such that the solution series is independent of $h$, a multiple of $h$-curves are plotted as shown in Figs. 2 and 3. The region where the distribution of $\dot{\beta}(0)$ and $\ddot{\beta}(1)$ versus $h$ is a horizontal line is known as the convergence region for the corresponding function.
It is easy to discover the valid region of $\hbar$, for example from Fig. 3 it is clear that acceptable region of $\hbar$ is $-0.6 \leq$ $\hbar \leq-0.3$, and from Fig. 3 the range for the acceptable values of $\hbar$ is $-0.5 \leq \hbar \leq-0.15$. Moreover, increasing the order of approximation, the range for the acceptable values of $\hbar$ increases (see Fig. 2).

\section{Result and discussion}

In Tables 1 and 2 a comparison between the numerical solution obtained by the present authors with HAM solution and those by Liabai and Simmonds [1] has been made for several dimensionless weight parameters, $\mu$, for the topcoordinate of the deformed cantilevered beamshell. Tip 


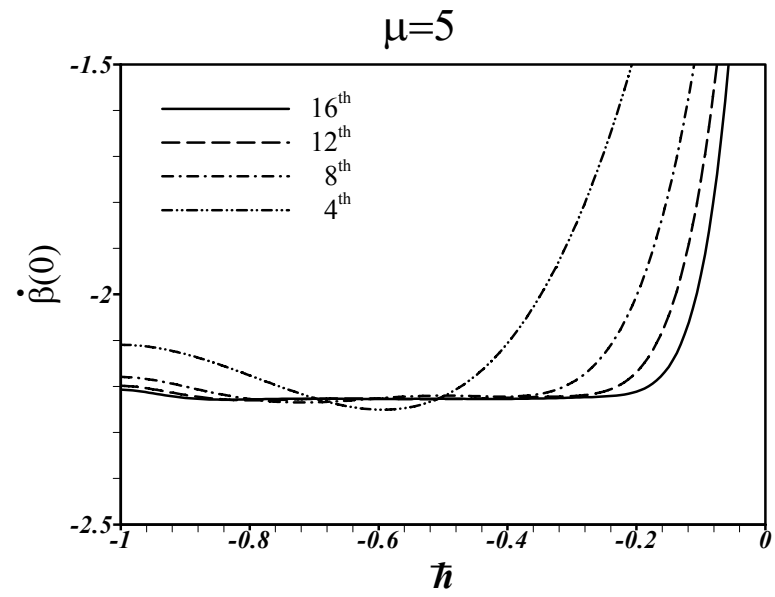

(a)

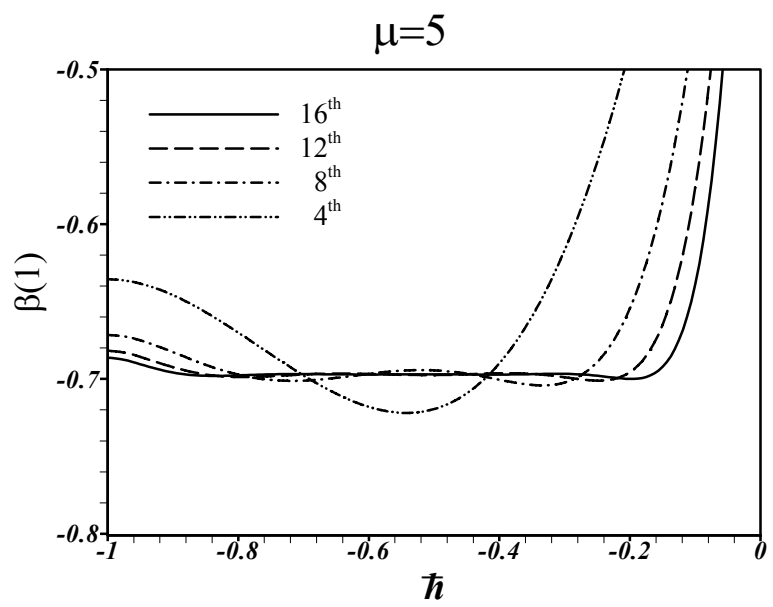

(b)

Figure 2. Comparison of convergence for different orders, $\mu=5$, (a) $\dot{\beta}(0)$, (b) $\beta(1)$.

vertical and horizontal deflection $(x(L), y(L))$ and slope are presented. It is clear that by increasing the weight dimensionless parameter, tip-deflection and slope increase.

It has been explained that $\hbar$ can be adjusted to get most accurate solution. Based on the h-curves (Fig. 2 and Fig. 3) it can be easily seen that, the common region for $\hbar$ is $-0.5 \leq \hbar \leq-0.3$ to have the same $\hbar$-value for all the solution. On the other hand, the interval of $\hbar$ can be increased by increasing the number of iterations in series solution. From the $\hbar$-curves the appropriate value of $\hbar$ is chosen; for example, from Fig. $3, \hbar=-0.3$ is taken for $\mu=10$.

It should be noted that when the weight of beam increases (i.e. weight dimensionless parameter $\mu$ takes larger values approaching $\infty$ ), the angle of beam tip approaches to $\pi / 2$. It should also be mentioned that in this paper the approx-
Table 1. Comparison of the HAM solution and numerical solution by Liabai and Simmonds [1] for tip rotation of a heavy elastica $(L=1)$.

\begin{tabular}{rcccc}
\hline$\mu$ & $\beta(1)$ by Ref $[1]$ & $\beta(1)$ by HAM & $\hbar$ & Relative Error $^{a}$ \\
\hline \hline 0.0 & 0.0000 & 0.0000 & -0.6 & 0.00000 \\
1.0 & -0.1651 & -0.1651 & -0.6 & 0.00009 \\
2.0 & -0.3216 & -0.3216 & -0.6 & 0.00002 \\
3.0 & -0.4635 & -0.4635 & -0.6 & 0.00010 \\
4.0 & -0.5886 & -0.5886 & -0.6 & 0.00001 \\
5.0 & -0.6970 & -0.6971 & -0.6 & 0.00021 \\
6.0 & -0.7904 & -0.7906 & -0.6 & 0.00022 \\
7.0 & -0.8708 & -0.8727 & -0.5 & 0.00218 \\
8.0 & -0.9401 & -0.9379 & -0.4 & 0.00235 \\
9.0 & -1.0002 & -1.0006 & -0.4 & 0.00042 \\
10.0 & -1.0526 & -1.0520 & -0.3 & 0.00060 \\
11.0 & -1.0985 & -1.0978 & -0.2 & 0.00059 \\
12.0 & -1.1389 & -1.1466 & -0.2 & 0.00675 \\
13.0 & -1.1747 & -1.1697 & -0.15 & 0.00429 \\
14.0 & -1.2065 & -1.1984 & -0.15 & 0.00675 \\
15.0 & -1.2348 & -1.2294 & -0.15 & 0.00434 \\
\hline
\end{tabular}

${ }^{a}$ Relative error defined as $\left|\left(\beta_{\text {HAM }}-\beta_{\text {Ref }[1]]}\right) / \beta_{\text {Ref. }[1]}\right|$

Table 2. Comparison of the HAM solution and numerical solution by Liabai and Simmonds [1] for tip deflection of a heavy elastica $(L=1)$.

\begin{tabular}{rrrrrrrr}
\hline$\mu$ & $y(1)$ by & $y(1)$ by & $x(1)$ by & $x(1)$ by & $\hbar$ & $\begin{array}{c}\text { R.E. }^{a} \\
\text { for } y\end{array}$ & $\begin{array}{c}\text { R.E. }^{a} \\
\text { for } x\end{array}$ \\
\hline \hline 0.0 & 0.0000 & 0.0000 & 1.0000 & 1.0000 & -0.60 & 0.000 & 0.000 \\
1.0 & -0.1235 & -0.1236 & 0.9912 & 0.9922 & -0.60 & 0.000 & 0.001 \\
2.0 & -0.2385 & -0.2387 & 0.9669 & 0.9679 & -0.60 & 0.001 & 0.001 \\
3.0 & -0.3396 & -0.3399 & 0.9315 & 0.9325 & -0.60 & 0.001 & 0.001 \\
4.0 & -0.4252 & -0.4254 & 0.8901 & 0.8910 & -0.60 & 0.001 & 0.001 \\
5.0 & -0.4959 & -0.4963 & 0.8467 & 0.8475 & -0.60 & 0.001 & 0.001 \\
6.0 & -0.5539 & -0.5544 & 0.8037 & 0.8045 & -0.60 & 0.001 & 0.001 \\
7.0 & -0.6015 & -0.6028 & 0.7628 & 0.7627 & -0.50 & 0.002 & 0.000 \\
8.0 & -0.6406 & -0.6400 & 0.7244 & 0.7264 & -0.40 & 0.001 & 0.003 \\
9.0 & -0.6731 & -0.6736 & 0.6890 & 0.6896 & -0.40 & 0.001 & 0.001 \\
10.0 & -0.7002 & -0.7003 & 0.6564 & 0.6575 & -0.30 & 0.000 & 0.002 \\
11.0 & -0.7231 & -0.7226 & 0.6264 & 0.6281 & -0.20 & 0.001 & 0.003 \\
12.0 & -0.7425 & -0.7450 & 0.5990 & 0.5958 & -0.20 & 0.003 & 0.005 \\
13.0 & -0.7591 & -0.7537 & 0.5739 & 0.5841 & -0.15 & 0.008 & 0.007 \\
14.0 & -0.7735 & -0.7673 & 0.5508 & 0.5567 & -0.15 & 0.007 & 0.008 \\
15.0 & -0.7860 & -0.7797 & 0.5296 & 0.5337 & -0.15 & 0.004 & 0.008 \\
\hline
\end{tabular}

${ }^{a}$ R.E.: Relative error defined as $\left|\left(y_{\text {HAM }}-y_{\text {Ref.[1] }}\right) / y_{\text {Ref. [1] }}\right|$

imation of the term $\cos (\beta)$ by Taylor series causes some small errors which can be considered negligible. If $\mu$ takes large values, the governing nonlinearity of the problem in Eq. 9 will appear, but even in this case HAM gives accurate results. According the obtained solution in comparison with the numerical results in Table 1 and 2, a remarkable accuracy even for large $\mu$ is observed. The HAM solution for slope and deformed configuration of cantilevered 


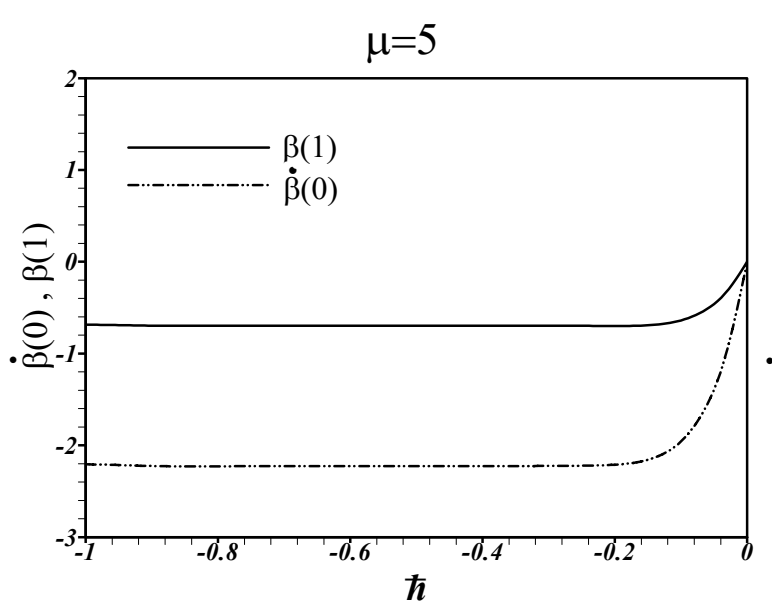

(a)

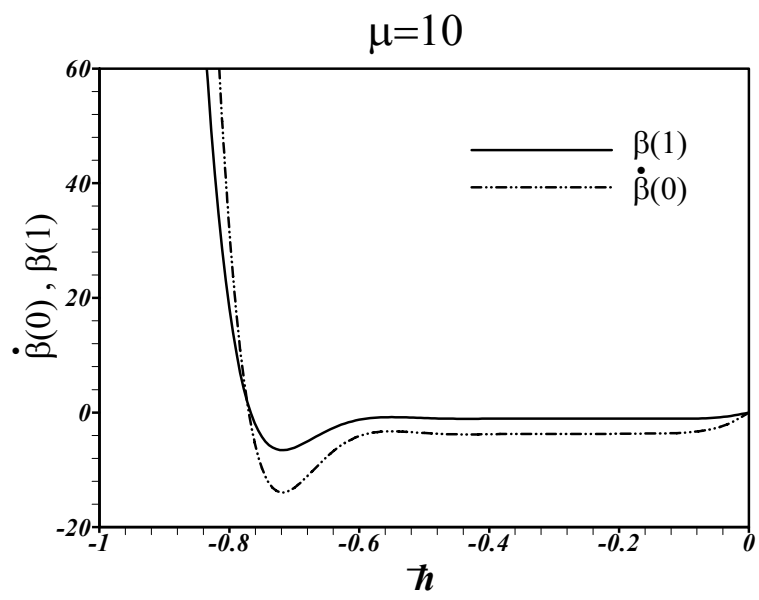

(c)

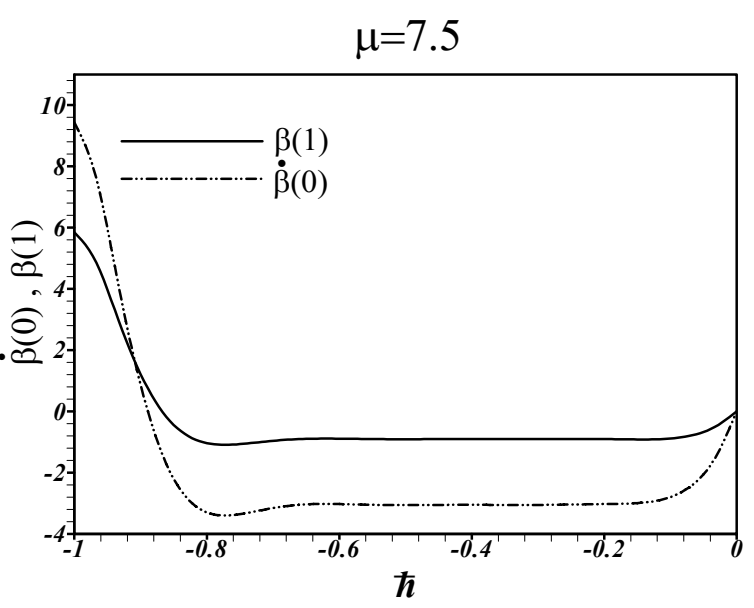

(b)

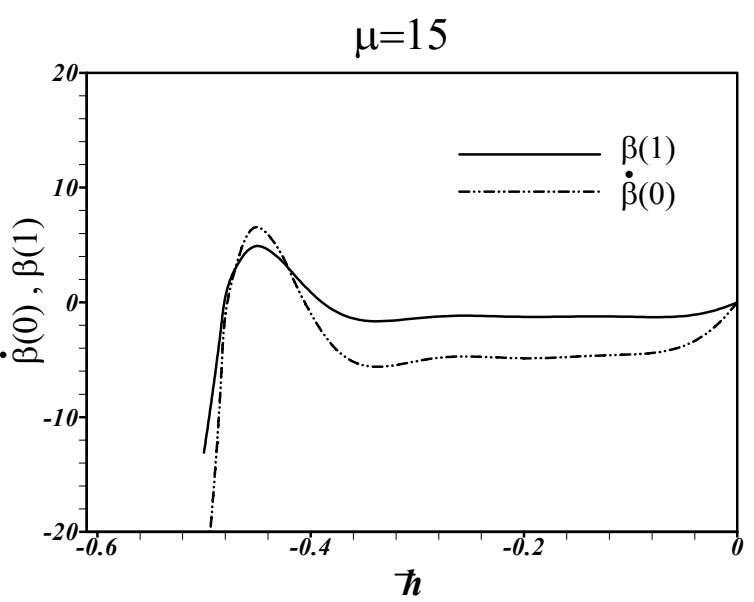

(d)

Figure 3. The $\hbar$ curve, (a) $\mu=5$, (b) $\mu=7.5$, (c) $\mu=10$ and (d) $\mu=15$. $20^{\text {th }}$-order approximation.

beamshell for different load cases are presented in Fig. 4 and Fig. 5 respectively, and the influence of dimensionless weight parameter is shown.

\section{Conclusions}

In this paper HAM was used to analyze a uniform cantilevered beamshell and an analytical solution was obtained for the first time. Compared to experimental and numerical results, a major advantage of analytical solutions is that they can cover a wide range of involved parameters, and it could be modified to a simple form that is useful for a particular purpose. An excellent rate of convergence and accurate results were demonstrated with a few iterations, and the results were in good agreement with the solution of previous studies. It can be concluded

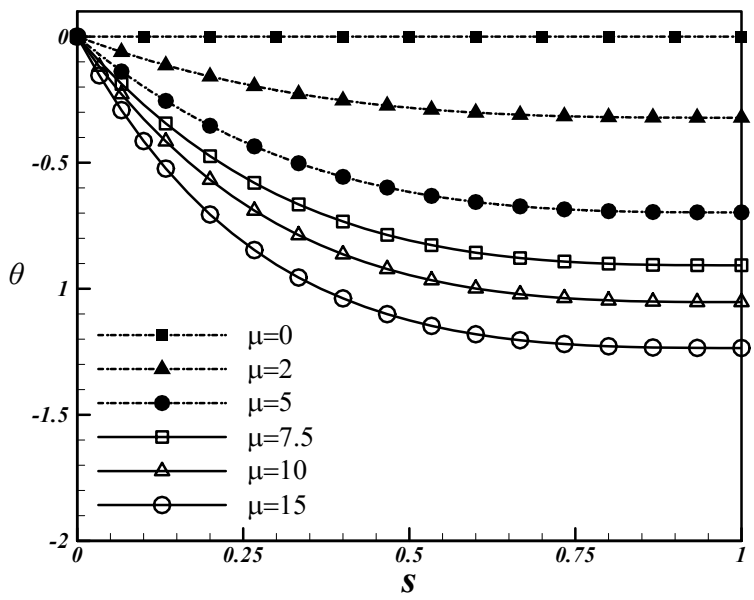

Figure 4. Effects of dimensionless weight parameter $\mu$ on angle of rotation. 


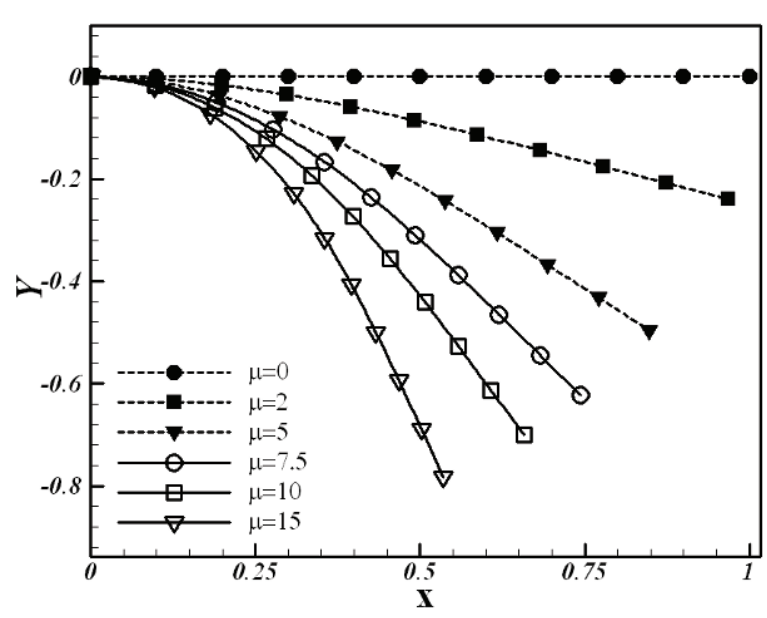

Figure 5. Effects of dimensionless weight parameter $\mu$ on position.

that the presented method is a suitable and efficient technique for the analysis of nonlinear and large deflection beamshell problems, and that it can be used for wide range of loads and lengths for beamshells with variable properties.

\section{Nomenclature}

$\begin{array}{ll}E_{b} & \text { Young's modulus } \\ F & \text { Stress function } \\ H & \text { Radial component of stress resultant vector } \\ H(s) & \text { Auxiliary function } \\ h & \text { Thickness of undeformed beamshell } \\ \hbar & \text { Auxiliary parameter } \\ l & \text { Moment of inertia } \\ L & \text { Undeformed length } \\ l & \text { Scalar external distributed couples } \\ M & \text { Bending moment } \\ m & \text { Mass per unit length } \\ P & \text { External surface normal load } \\ R_{m}\left(U_{m-1}\right) & \text { Reminder term } \\ S & \text { Independent dimensionless parameter } \\ V & \text { Component of stress resultant in the y-direction } \\ V & \text { Velocity } \\ x & \text { Cartesian coordinate } \\ \bar{x} & \text { x-Position } \\ y & \text { Cartesian coordinate } \\ \bar{y} & \text { y-Position } \\ \beta & \text { Rotational deformation } \\ \sigma & \text { Shell coordinate } \\ \lambda & \text { Stretches } \\ \mu & \text { Dimensionless weight parameter }\end{array}$

\section{References}

[1] Liabai A., Simmonds J.G. (1998): The nonlinear theory of elastic shells, Cambridge university press

[2] Hummel F.H., Morton W.B., On the Large bending of thin flexible strips and the measurement of their elasticity, Philos. Mag., 1927, 4, 384-357

[3] Bickley W.G., The heavy elastica, Philos. Mag., 1934, 17, 603-622

[4] Rohde F.V., Large deflections of a cantilever beam with a uniform distributed load, Q. Appl. Math., 1953, 11, 337-338.

[5] Wang T.M., Non-linear bending of beams with uniformly distributed loads, Int. J. Nonlinear Mech., 1969, 4, 389-395

[6] Van der Heijden, On the influence of the bending stiffness in cable analysis, Proc. Kon. Ned. Ak. Wet., 1973, 76, 217-229

[7] Simmonds J.G., Jr. Mann J.E. (1986): A First Look at Perturbation Theory. Krieger, New York

[8] Holden J.T., On the finite deflections of thin beams, Int. J. Solids. Struct., 1972, 8, 133-135

[9] Wang C.Y., Folding of elastica: similarity solutions. J. Appl. Mech., 1981, 48, 199-20

[10] Wang C.Y., Large deflection of an inclined cantilever with an end load. tem5 Wang T.M., Non-linear bending of beams with uniformly distributed loads, Int. J. Nonlinear Mech., 1981, 16, 155-164

[11] Wang C.Y., Lifting a heavy elastic sheet or rod from an incline, Int. J. Mech. Sci., 1983, 25, 851-858

[12] Liao S.J. (1992): The proposed homotopy analysis technique for the solution of nonlinear problems, Shanghai Jiao Tong University

[13] Liao S.J. (2003) :Beyond perturbation: introduction to homotopy analysis method, Boca Raton: Chapman and Hall/CRC Press

[14] Wanga Ji., Chena J.K., Liao S.J., J. Comp. Appl. Math., 2008, 212, 320-330

[15] Liao S.J., An approximate solution technique which does not depend upon small parameters: a special example, Int. J. Nonlinear Mech., 1995, 30, 371-380

[16] Kimiaeifar A., Saidi A.R., Bagheri G.H., Rahimpour M., Domairry D.G., Analytical solution for Van der Pol-Duffing oscillators, Chaos, Solitons and Fractals, 2009, 42, 2660-2666

[17] Kimiaeifar A., Mohebpour S.R., Sohouli A.R., Domairry G.M, Davodi A. G., Analytical Solution for Large Deflections of a Cantilever Beam under Nonconservative Load Based on Homotopy Analysis Method, J. Numer. Meth. Part. Differ. Equat., 2011, 27, 541-553 
[18] Chen Y.M., Liu J.K., Uniformly valid solution of limit cycle of the Duffing-van der Pol equation, Mech. Res. Comm., 2009, 36, 845-85

[19] Kimiaeifar A., An analytical approach to investigate the response and stability of Van der Pol-MathieuDuffing oscillators under different excitation functions, J. Math. Meth. Appl. Sci., 2010, 33, 1571-1577
[20] Ibsen L.B., Barari A., Kimiaeifar A., Analysis of Strongly Nonlinear Oscillation Systems Using He's Max-Min Method and Comparison with Homotopy Analysis Method and Energy Balance, Sadhana, 2010, 35, 433-448 